\title{
Role of RNF213 polymorphism in defining quasi-moyamoya disease and definitive moyamoya disease
}

\author{
Eitaro Ishisaka, MD, ${ }^{1}$ Atsushi Watanabe, MD, PhD, ${ }^{2,3}$ Yasuo Murai, MD, PhD, ${ }^{1}$ \\ Kazutaka Shirokane, MD, PhD, ${ }^{4}$ Fumihiro Matano, MD, PhD, ${ }^{1}$ Atsushi Tsukiyama, MD, PhD, ${ }^{1}$ \\ Eiichi Baba, MD, ${ }^{1}$ Shunsuke Nakagawa, MD, PhD, ${ }^{1}$ Tomonori Tamaki, MD, PhD, ${ }^{4}$ \\ Takayuki Mizunari, MD, PhD, ${ }^{5}$ Rokuya Tanikawa, MD, ${ }^{6}$ and Akio Morita, MD, PhD ${ }^{1}$
}

\begin{abstract}
'Department of Neurological Surgery, Nippon Medical School, Bunkyo-ku, Tokyo; ${ }^{2}$ Division of Clinical Genetics, Kanazawa University Hospital, Kanazawa, Ishikawa; ${ }^{3}$ Support Center for Genetic Medicine, Kanazawa University Hospital, Kanazawa, Ishikawa; ${ }^{4}$ Department of Neurosurgery, Nippon Medical School, Tama-Nagayama Hospital, Tama, Tokyo; ${ }^{5}$ Department of Neurosurgery, Chiba Hokuso Hospital, Nippon Medical School, Chiba; and ${ }^{6}$ Department of Neurosurgery, Teishinkai Hospital, Sapporo, Hokkaido, Japan
\end{abstract}

OBJECTIVE Quasi-moyamoya disease (QMMD) is moyamoya disease (MMD) associated with additional underlying diseases. Although the ring finger protein 213 (RNF213) c.14576G>A mutation is highly correlated with MMD in the Asian population, its relationship to QMMD is unclear. Therefore, in this study the authors sought to investigate the RNF213 c.14576G >A mutation in the genetic diagnosis and classification of QMMD.

METHODS This case-control study was conducted among four core hospitals. A screening system for the RNF213 c.14576G $>$ A mutation based on high-resolution melting curve analysis was designed. The prevalence of RNF213 c.14576G>A was investigated in 76 patients with MMD and 10 patients with QMMD.

RESULTS There were no significant differences in age, sex, family history, and mode of onset between the two groups. Underlying diseases presenting in patients with QMMD were hyperthyroidism $(n=6)$, neurofibromatosis type $1(n=2)$, Sjögren's syndrome $(\mathrm{n}=1)$, and meningitis $(\mathrm{n}=1)$. The RNF213 c.14576G>A mutation was found in 64 patients $(84.2 \%)$ with MMD and 8 patients (80\%) with QMMD; no significant difference in mutation frequency was observed between cohorts.

CONCLUSIONS There are two forms of QMMD, one in which the vascular abnormality is associated with an underlying disease, and the other in which MMD is coincidentally complicated by an unrelated underlying disease. It has been suggested that the presence or absence of the RNF213 c.14576G>A mutation may be useful in distinguishing between these disease types.

https://thejns.org/doi/abs/10.3171/2021.5.FOCUS21182

KEYWORDS quasi-moyamoya disease; RNF213 c.14576G>A mutation; vascular disorders

$\mathrm{M}$ OYAMOYA disease (MMD) is a cryptogenic, progressive cerebral vascular occlusion that frequently occurs in the Asian population. Cerebral angiography shows stenosis or occlusion at the terminal portion of the internal carotid arteries and abnormal vascular networks around the occlusion. Quasi-moyamoya disease (QMMD) is MMD associated with certain underlying diseases. According to current diagnostic criteria, patients known to have MMD and underlying diseases receive a diagnosis of QMMD, whereas patients with MMD and a coincidental unrelated underlying disease are excluded. ${ }^{1}$ Although treatments available for QMMD are similar to those for MMD, certain medical treatments are effective in some patients with hyperthyroidism or an underlying autoimmune disease..$^{2-4}$ Furthermore, advances in the treatment of underlying diseases could also affect the pathophysiology of QMMD. Therefore, distinguishing definitive QMMD caused by an underlying disease from the concurrent appearance of an underlying disease and MMD will enable more accurate diagnosis and selection of appropriate treatment strategies.

Recent advances in gene analysis technology have benefited the field of cerebrovascular disorders. The identification of the role of genetic factors in cerebrovascular

ABBREVIATIONS MMD = moyamoya disease; NF1 = neurofibromatosis type $1 ; \mathrm{PCR}=$ polymerase chain reaction; $R N F 213=$ ring finger protein $213 ;$ SAG = small amplicon genotyping; $\mathrm{QMMD}=$ quasi-MMD. 
disorders contributes not only to the development of new methods of molecular-targeted therapy but also to better genetic differential diagnosis, appropriate risk assessment, and onset prediction. Furthermore, better understanding these genetic factors might lead to the establishment of new diagnostic criteria and disease concepts. In recent years, ring finger protein 213 (RNF213) has been identified as a disease-susceptibility gene for MMD, and a single missense mutation in RNF213 (c.14576G >A, p.R4859K, rs112735431) is frequently found in studies of East Asian populations..$^{5-7}$ However, the relationship between this gene mutation and QMMD is unclear as it has received little attention, and the findings among those few studies vary considerably. Thus, the purpose of the current study was to investigate the role of the RNF213 c. $14576 \mathrm{G}>\mathrm{A}$ mutation in the genetic diagnosis and classification of QMMD by examining the mutation frequency in patients with MMD and those with QMMD.

\section{Methods \\ Patients}

Our case-control study was conducted among four core hospitals. Genetic analysis of the RNF213 c.14576G>A mutation was performed in patients with MMD or QMMD who visited one of these hospitals between August 2014 and August 2018 and agreed to inclusion in the study. This study was approved by the ethics committee of each hospital and the ethics committee of Nippon Medical School. Written informed consent was acquired from all participants. The following information was obtained from all patients: sex, age at diagnosis, family history, onset symptoms, underlying diseases, and the presence or absence of lifestyle diseases.

\section{Diagnosis}

We confirmed that the diagnoses of MMD and QMMD complied with the respective diagnostic criteria detailed in the Japanese guidelines outlined in "Recommendations for the Management of Moyamoya Disease: A Statement from Research Committee on Spontaneous Occlusion of the Circle of Willis (Moyamoya Disease) [2nd Edition]."1

Patients with underlying diseases or stenotic occlusion lesions due to arteriosclerosis were excluded from an MMD diagnosis. MMD diagnosis was made using imaging procedures, including MRI, MRA, and cerebrovascular angiography. Some patients with MMD were diagnosed using MRI/MRA according to the criteria that stenosis or occlusion is found in the area focusing on the end of the intracranial internal carotid arteries and that abnormal vascular networks are found in the basal ganglia, as observed on MRA images. Most patients were diagnosed using cerebrovascular angiography according to the criteria that stenosis or occlusion is found in the area focusing on the intracranial internal carotid arteries and that abnormal vascular networks (i.e., moyamoya blood vessels) are observed in the vicinity of arterial phases.

Underlying diseases in patients with QMMD were identified based on medical history obtained from the patient or a family member and medical records. Family history was obtained from either the patient or a family member.
In the current study, a diagnosis of QMMD was defined as stenosis or occlusion observed at the end of the internal carotid arteries and in the vicinity of the anterior and middle cerebral arteries and the involvement of abnormal vascular networks. Patients with unilateral lesions who had underlying diseases were included in the QMMD group.

The following conditions have been reported as underlying diseases: arteriosclerosis, autoimmune diseases, meningitis, von Recklinghausen's disease, brain tumor, Down syndrome, head injuries, radiotherapy, hyperthyroidism, and some other diseases. ${ }^{1}$ In this study, arteriosclerosis was excluded as an underlying disease because of its known association with the RNF213 c.14576G $>\mathrm{A}$ mutation. ${ }^{8}$

\section{DNA Extraction and RNF213 Genotyping}

After written informed consent was obtained from included patients, peripheral blood samples were collected. Genomic DNA extraction from blood was performed using a GENOMIX kit (Talent). RNF213 c.14576G $>\mathrm{A}$ (exon 61) genotype screening was performed by small amplicon genotyping (SAG) based on high-resolution melting curve analysis ${ }^{9}$ and confirmed by Sanger sequencing. Polymerase chain reaction (PCR) primers for $\mathrm{c} .14576 \mathrm{G}>\mathrm{A}$ were designed to flank the mutation leaving only a single base, including the mutation between the primers. The forward primer used was 5'-GCAAGTTGAATACAGCTC CATCA-3', and the reverse primer was 5'-TGTGCTT GCTGAGGAAGCCT-3'. PCR conditions were as follows: initial denaturation at $95^{\circ} \mathrm{C}$ for 2 minutes, followed by 45 cycles at $94^{\circ} \mathrm{C}$ for 30 seconds and annealing at $67^{\circ} \mathrm{C}$ for 30 seconds. After PCR, high-resolution melting curve analysis was performed in 96 well plates using a LightScanner (Idaho Technology Inc.), during which data were collected from $55^{\circ} \mathrm{C}$ to $97^{\circ} \mathrm{C}$ at a ramp rate of $0.101^{\circ} \mathrm{C}$ per second.

\section{Data Analysis}

Comparative analyses on age at onset, sex, onset symptom, family history, and frequency of the RNF213 c.14576G $>$ A mutation were performed between the MMD and QMMD cohorts. In addition, univariate analyses were performed to compare age, sex, mode of onset, and risk factors for arteriosclerosis (smoking, hypertension, diabetes mellitus, dyslipidemia, and ischemic heart disease) between patients with and those without gene mutations. All values are expressed as mean $\pm \mathrm{SE}$ or median and IQR. The Mann-Whitney U-test and the Fisher's exact test were used to assess statistical significance due to the heterogeneity and small sample size. All analyses were performed at a significance level of $\mathrm{p}<0.05$ using a commercially available software package (JMP Pro 13, SAS Institute Inc.).

\section{Results}

The two RNF213 c.14576G >A genotypes, namely c.14576GG (wt/wt) and c.14576AG (mut/wt), were determined by a modified SAG method for all patients in our study (Fig. 1).

A total of 86 patients (76 with MMD and 10 with QMMD) were included in the study. Patient characteris- 

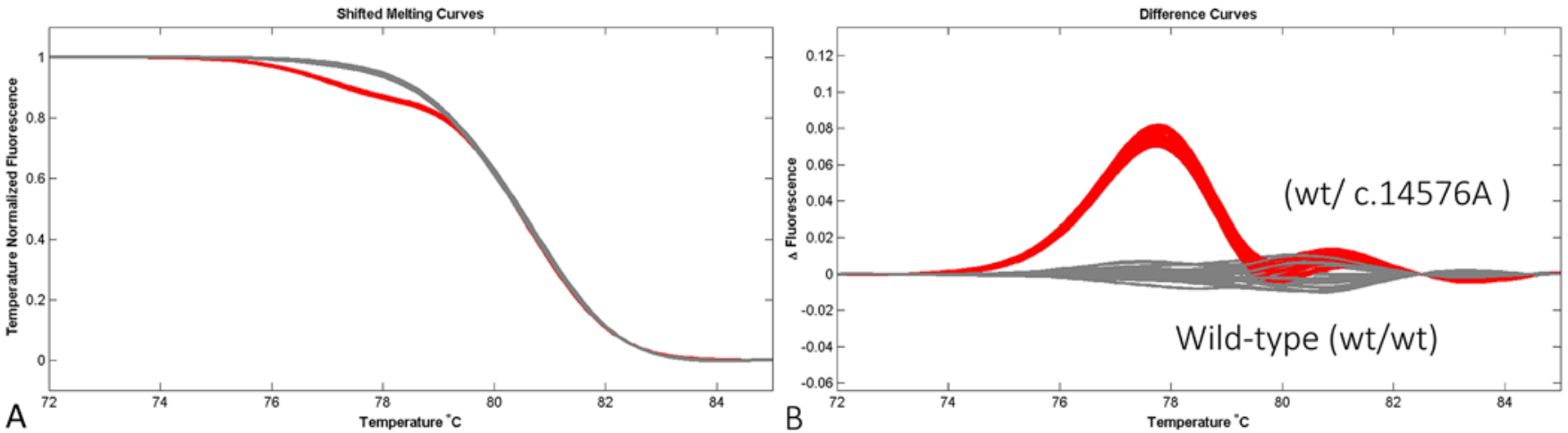

FIG. 1. Identification of the c.14576G>A (exon 61) mutation in RNF213 by the SAG method. A: Normalized fluorescence plots. B: The corresponding fluorescence difference plots. Wild-type (wt, c.14576G/wt) samples are in gray and heterozygous samples $(\mathrm{wt} / \mathrm{c} .14576 \mathrm{~A})$ are in red. The two genotypes were clearly distinguishable in the SAG method.

tics are presented in Table 1. The mean age at onset was 35 years for all patients, 34 years (range 1-77 years) for patients with MMD, and 43.4 years (range 20-62 years) for patients with QMMD. Our study cohort consisted of 61 females (55 with MMD and 6 with QMMD) and 25 males (21 with MMD and 4 with QMMD). In addition, positive family history was found in $15.8 \%$ of patients with MMD $(12 / 76)$ and $10 \%$ of patients with QMMD (1/10).

The onset symptoms of MMD in our cohort were cerebral ischemic disease in 48 patients, hemorrhage in 11 patients, headache in 7 patients, no symptoms in 4 patients, and other onset type in 6 patients. The onset symptoms of QMMD in our cohort were ischemia in 6 patients, hemorrhage in 0 patients, headache in 2 patients, no symptoms in 1 patient, and other onset type in 1 patient. We found no significant differences in age, sex, family history, and onset symptoms between the MMD and QMMD groups.

The RNF213 c.14576G>A mutation was found in 64 patients (84.2\%) in the MMD group and 8 patients (80\%) in the QMMD group; there was no significant difference in mutation frequency between the two groups $(p=0.584)$. In addition, subgroup analysis showed no significant differences in age, sex, family history, and onset symptoms between patients with MMD and patients with QMMD based on RNF213 c. $14576 \mathrm{G}>\mathrm{A}$ mutation status.

We found the following underlying diseases among the patients with QMMD in our group: hyperthyroidism in 6 patients, neurofibromatosis type 1 (NF1) in 2 patients, Sjögren's syndrome in 1 patient, and meningitis in 1 patient. The RNF213 c.14576G $>$ A mutation was found in 5 patients with hyperthyroidism, 1 patient with NF1, 1 patient with Sjögren's syndrome, and 1 patient with meningitis (Table 2).

The results of comparisons of age, sex, mode of onset, clinical findings, family history, and risk factors for arteriosclerosis (smoking, hypertension, diabetes mellitus, dyslipidemia, and ischemic heart disease) between patients with and those without RNF213 gene mutations are presented in Table 3 . None of the parameters showed significant differences. Only a family history of MMD tended to be associated with gene mutations $(\mathrm{p}=0.0928)$.

\section{Discussion}

In this study, 80\% of patients with QMMD carried the RNF213 c.14576G >A mutation. As the prevalence was not significantly different from that found in patients with MMD, we found that our MMD and QMMD cohorts had similar rates of gene mutation. Autoimmune diseases were the most common underlying diseases in patients with QMMD in this study, as cases of atherosclerosis were excluded. Furthermore, the prevalence of gene mutation in patients with QMMD in this study was extremely high compared with that found in previous studies on gene mutation in patients with QMMD. This finding indicates that many patients with conventional MMD who coincidentally have concurrent underlying disease were included as patients with QMMD in our study. Based on these findings, we speculate that among the patients diagnosed with QMMD according to current diagnostic criteria, patients with this gene mutation are classified as having definitive

TABLE 1. Demographic characteristics and results of genetic analysis of the RNF213 p.R4810K variant in patients

\begin{tabular}{|c|c|c|c|}
\hline & MMD Group & QMMD Group & $p$ Value \\
\hline No. of patients & 76 & 10 & \\
\hline Mean age, yrs (range) & $34(1-77)$ & $43.4(20-62)$ & 0.12 \\
\hline Female sex & $55(72.4)$ & $6(60)$ & 0.47 \\
\hline Family history of MMD & $12(15.8)$ & $1(10)$ & 0.70 \\
\hline \multicolumn{3}{|l|}{ Onset symptom } & \multirow{6}{*}{0.59} \\
\hline Ischemia (TIA or CI) & $48(63.2)$ & $6(60)$ & \\
\hline Hemorrhage & $11(14.5)$ & $0(0)$ & \\
\hline Headache & $7(9.2)$ & $2(20)$ & \\
\hline Other & $6(7.9)$ & $1(10)$ & \\
\hline Asymptomatic & $4(5.3)$ & $1(10)$ & \\
\hline $\begin{array}{l}\text { Occurrence of RNF213 } \\
\text { c. } 14576 \mathrm{G}>\mathrm{A}\end{array}$ & $64(84.2)$ & $8(80)$ & $>0.99$ \\
\hline
\end{tabular}

$\mathrm{Cl}=$ cerebral infarction; $\mathrm{TIA}=$ transient ischemic attack.

Values represent the number of patients (\%) unless indicated otherwise. 
TABLE 2. Clinical characteristics of patients with QMMD in this study

\begin{tabular}{ccccccc}
\hline Patient No. & Sex & Age Group & Coexisting Disease & Onset Symptom & Family History & RNF213 c.14576G>A \\
\hline 1 & M & Middle age & Hyperthyroidism & Headache & No & Yes \\
\hline 2 & M & Middle age & Hyperthyroidism & Asymptomatic & Yes & Yes \\
\hline 3 & F & Young adult & Hyperthyroidism & Ischemic stroke & No & Yes \\
\hline 4 & F & Young adult & Hyperthyroidism & Ischemic stroke & No & Yes \\
\hline 5 & F & Middle age & Hyperthyroidism & Ischemic stroke & No & No \\
\hline 6 & F & Young adult & NF1 & Ischemic stroke & No & Yes \\
\hline 7 & F & Middle age & Sjögren's syndrome & Ischemic stroke & No & Yes \\
\hline 8 & M & Young adult & Meningitis & Ischemic stroke & No & Yes \\
\hline 9 & M & Middle age & NF1 & Other & No & No \\
\hline 10 & F & Middle age & Hyperthyroidism & Asymptomatic & No & Yes \\
\hline
\end{tabular}

MMD complicated with a coincidental underlying disease, whose vascular abnormalities are unlikely to be improved by treatment of the underlying disease. In contrast, for patients with QMMD lacking this gene mutation, vascular abnormalities might have occurred due to the underlying

TABLE 3. Factors associated with and without the RNF213 p.R4810K variant

\begin{tabular}{|c|c|c|c|c|}
\hline & Total & $\begin{array}{c}R N F 213 \\
\text { c. } 14576 \mathrm{G}>\mathrm{A} \\
\text { (yes) }\end{array}$ & $\begin{array}{c}R N F 213 \\
\text { c. } 14576 \mathrm{G}>\mathrm{A} \\
\text { (no) }\end{array}$ & $\begin{array}{c}p \\
\text { Value }\end{array}$ \\
\hline $\begin{array}{l}\text { Total no. of } \\
\text { patients }\end{array}$ & 86 & 72 & 14 & \\
\hline $\begin{array}{l}\text { Patients w/ } \\
\text { QMMD }\end{array}$ & 10 & 8 & 2 & 0.5120 \\
\hline $\begin{array}{l}\text { Age at diagnosis, } \\
\text { yrs }^{*}\end{array}$ & & & & $0.9720^{*}$ \\
\hline Mean \pm SD & $35.13 \pm 1.93$ & $35.12 \pm 2.17$ & $35.21 \pm 4.18$ & \\
\hline Median (IQR) & $37.5(28.75)$ & 37 (31.25) & $40(19.5)$ & \\
\hline $\begin{array}{l}\text { Age at diagnosis } \\
>16 \mathrm{yrs}\end{array}$ & 66 & 54 & 12 & $0.3133 \dagger$ \\
\hline $\begin{array}{l}\text { Hemorrhage } \\
\text { onset }\end{array}$ & 11 & 11 & 0 & $0.1235 \dagger$ \\
\hline Ischemic onset & 54 & 43 & 11 & $0.1506 \dagger$ \\
\hline $\begin{array}{l}\text { Ischemia or hem- } \\
\text { orrhage onset }\end{array}$ & 62 & 52 & 10 & $0.5916 \dagger$ \\
\hline Male sex & 25 & 21 & 4 & $0.6195 \dagger$ \\
\hline Smoking & $\begin{array}{c}13 \\
\text { (2 unknown) }\end{array}$ & 11 & 2 & $0.6285 \dagger$ \\
\hline Hypertension & 37 & 32 & 5 & $0.3826 \dagger$ \\
\hline Dyslipidemia & 22 & 20 & 2 & $0.2414 \dagger$ \\
\hline Diabetes mellitus & 11 & 11 & 0 & $0.1235 \dagger$ \\
\hline $\begin{array}{l}\text { Ischemic heart } \\
\text { disease }\end{array}$ & 5 & 4 & 1 & $0.5982 \dagger$ \\
\hline $\begin{array}{l}\text { Family history of } \\
\text { MMD }\end{array}$ & $\begin{array}{c}13 \\
\text { (2 unknown) }\end{array}$ & 13 & 0 & $0.0928 \dagger$ \\
\hline
\end{tabular}

* Mann-Whitney U-test.

$\dagger$ Fisher's exact test. disease, and thus, treatment of the underlying disease may be effective in improving QMMD.

According to the definitions used in this study, patients with QMMD and any underlying disease all received a diagnosis of QMMD regardless of whether there was a causal relationship between the underlying disease and vascular abnormalities. ${ }^{1}$ Therefore, patients with intracranial vascular abnormalities due to the underlying disease are considered together with those patients who coincidentally have MMD and an independent underlying disease. Thus, a diagnosis of idiopathic MMD is not sufficiently distinguishable from secondary MMD, and QMMD is inherently not the same as secondary MMD.

Treatment modalities available for QMMD are similar to those available for MMD; however, certain medical treatments are effective for some patients with underlying diseases such as hyperthyroidism or autoimmune diseases. ${ }^{2-4}$

Because the underlying diseases affect vascular structures, their treatment can lead to improvement in vascular abnormalities. Therefore, advances in treatment strategies for underlying diseases may lead to more patients with QMMD experiencing improvement as a result of nonsurgical medical treatment in the future. However, there is currently no method to preoperatively identify those patients with a direct causal relationship between an underlying disease and vascular abnormalities; therefore, indications for surgery and the appropriate timing of interventions are not clear. ${ }^{1}$

Moreover, although the prediction of factors of pathological conditions, such as progression of vascular abnormalities, onset time, presence or absence of aggravation, and differences in phenotype, is important in patient management, no unified view has yet been derived regarding QMMD because of the diversity of underlying diseases.-11 For example, in clinical practice, we found a high prevalence of patients with QMMD and hyperthyroidism whose vascular abnormalities were not improved by the treatment of hyperthyroidism; such patients with QMMD were also found in the current study. ${ }^{4,9,12}$ It is inferred that there are cases of secondary MMD with a causal relationship to the underlying disease as well as cases of idiopathic MMD that are poorly associated with the underlying disease in QMMD, both of which are associated with the same un- 
TABLE 4. Review of previous studies

\begin{tabular}{|c|c|c|c|c|c|c|}
\hline Authors \& Year & $\begin{array}{l}\text { No. of } \\
\text { Patients }\end{array}$ & $\begin{array}{l}\text { Female } \\
\text { Sex, n (\%) }\end{array}$ & $\begin{array}{l}\text { Age } \\
\text { (yrs) }\end{array}$ & Population & Coexisting Disease & $\begin{array}{c}\text { Rate of Occurrence of RNF213 } \\
\text { p.R4810K Variant, } \mathrm{n}(\%)\end{array}$ \\
\hline $\begin{array}{l}\text { Miyawaki et al., } \\
2015^{15}\end{array}$ & 9 & $8(88.9)$ & 34.2 & Japanese & $\begin{array}{l}3 \text { radiotherapy, } 2 \text { hyperthyroidism, } 1 \text { Turner } \\
\text { syndrome, } 1 \text { meningitis, } 1 \text { Behçet disease, } \\
1 \text { idiopathic pachymeningitis }\end{array}$ & $0(0)$ \\
\hline Chong et al., $2015^{18}$ & 1 & $1(100)$ & 2 & Korean & 1 Down syndrome & $1(100)$ \\
\hline Phi et al., $2016^{17}$ & 16 & $10(62.5)$ & 5.5 & Korean & $16 \mathrm{NF1}$ & $3(18.7)$ \\
\hline $\begin{array}{l}\text { Morimoto et al., } \\
2016^{16}\end{array}$ & 18 & $14(77.8)$ & 34.3 & Japanese & $\begin{array}{l}4 \text { NF1, } 4 \text { hyperthyroidism, } 2 \text { Down syndrome, } \\
2 \text { Kawasaki disease, } 2 \text { rheumatoid arthritis, } \\
1 \text { Noonan syndrome, } 1 \text { systemic lupus ery- } \\
\text { thematosus, } 1 \text { autoimmune pancreatitis, } 1 \\
\text { Sjögren's syndrome \& Hashimoto thyroiditis }\end{array}$ & $12(66.7)$ \\
\hline Zhang et al., $2017^{19}$ & 42 & $24(57.1)$ & 34.9 & Chinese & $\begin{array}{l}22 \text { arteriosclerosis, } 13 \text { autoimmune diseases, } \\
3 \mathrm{NF1}, 2 \text { brain neoplasm/radiotherapy, } 1 \\
\text { Down syndrome, } 1 \text { thalassemia }\end{array}$ & $5(11.9)$ \\
\hline Nomura et al., $2019^{20}$ & 15 & $15(100)$ & 32 & Japanese & 15 hyperthyroidism & $8(53.3)^{*}$ \\
\hline Current study & 10 & $6(60)$ & 35 & Japanese & $\begin{array}{l}6 \text { hyperthyroidism, } 2 \text { NF1, } 1 \text { Sjögren's syn- } \\
\text { drome, } 1 \text { meningitis }\end{array}$ & $8(80)$ \\
\hline
\end{tabular}

* Includes rare and low-frequency missense variants in RNF213 other than the p.R4810K variant.

derlying disease. Thus, distinguishing MMD secondary to the underlying disease is important for treatment selection, in addition to better understanding the disease as well as its pathophysiology.

To better discriminate between MMD and QMMD, genetic diagnosis may be informative. A missense mutation in $R N F 213$ (c.14576G>A, p.R4859K, rs112735431) is highly correlated with MMD. The mutation is found at a high frequency in patients with MMD belonging to East Asian populations; approximately $80 \%$ of Japanese patients with MMD have this mutation, indicating a high correlation with clinical phenotypes such as early onset and disease exacerbation. ${ }^{5}$ In our study, the RNF213 c. $14576 \mathrm{G}>\mathrm{A}$ mutation was found in $84.2 \%$ of patients with MMD, a frequency which is similar to that found in previous reports..$^{6,713}$

Furthermore, this finding demonstrates the validity of using the SAG approach by high-resolution melting curve analysis for the identification and genotyping of RNF213 c. $14576 \mathrm{G}>\mathrm{A}$ mutation in our cohorts. This method is a convenient one-step, single-tube method to detect specific mutations, and it is faster, simpler, and lower in cost compared with other approaches requiring separation or labeled probes..$^{14}$

Data from previous reports and the current study are summarized in Table 4. In previous studies, the percentage of patients with QMMD and the $R N F 213$ c.14576G $>\mathrm{A}$ mutation varied considerably as follows: $0 \%(0 / 9)$ in the study by Miyawaki et al., ${ }^{15} 66.7 \%$ (12/18) in Morimoto et al., ${ }^{16} 18.7 \%$ (3/16) in Phi et al., ${ }^{17} 100 \%$ (1/1) in Chong et al., ${ }^{18} 11.9 \%(5 / 42)$ in Zhang et al., ${ }^{19}$ and $53.3 \%(8 / 15)$ in Nomura et al..$^{20}$ In comparison, we found that $80 \%(8 / 10)$ of our patients with QMMD had the mutation, indicating a high frequency. The average frequency reported in all previous reports was $37.6 \%$ (35/93). ${ }^{15-20}$ In addition, mutation frequency varied according to the underlying disease as follows: $48 \%(12 / 25)$ of patients had NF1, 48.5\% (16/33) had hyperthyroidism, $13.6 \%$ (3/22) had arteriosclerosis, $100 \%(3 / 3)$ had rheumatism, 33.3\% (1/3) had Down syndrome, and $0 \%(0 / 3)$ had undergone radiotherapy.

The differences in these results may be related to the inclusion and exclusion of underlying diseases with a high prevalence, such as hyperthyroidism and arteriosclerosis. Although a consistent definition of QMMD was applied to all studies, those by Phi et al., ${ }^{17}$ Chong et al. ${ }^{18}$ and Nomura et al. ${ }^{20}$ were limited to a single underlying disease; moreover, Morimoto et al. ${ }^{16}$ and Miyawaki et al. ${ }^{15}$ excluded arteriosclerosis as an underlying disease, whereas Zhang et al. ${ }^{19}$ included all underlying diseases. Specifically, patients with QMMD accompanied by hyperthyroidism tended to have a high positive rate of variants. Hyperthyroidism has a high prevalence, which suggests that there are many patients with conventional MMD who coincidentally have concurrent hyperthyroidism. Therefore, the current diagnostic criteria by which all patients are classified as having QMMD does not fully encompass the true disease condition with respect to the underlying disease such as hyperthyroidism. Thus, it confounds efforts to accurately determine the best treatment strategy and predict therapeutic effects. The considerable variation found among previous reports suggests that patients with a definitive QMMD diagnosis secondary to the underlying disease, and patients with a diagnosis of independent and conventional MMD, are grouped with patients diagnosed with QMMD.

Function analysis has demonstrated that RNF213 is implicated in angioplasty; however, the mechanism has not yet been clarified. In addition, as many gene carriers are patients with atherosclerotic lesions, pulmonary hypertension, or cardiovascular disease, as well as MMD, it is necessary to investigate the association between the RNF213 c.14576G $>$ A mutation and each underlying disease group associated with QMMD in the future. ${ }^{21-23}$ 
The RNF213 c.14576G>A mutation is found in $80 \%$ of Japanese patients with MMD. In contrast, it has been reported that the mutation is seldom found in patients with MMD from other ethnicities such as European populations. ${ }^{24}$ The reason for these population-specific differences is not known, and a number of factors related to the $R N F 213$ c. $14576 \mathrm{G}>\mathrm{A}$ mutation, such as function, distribution, and effect, require additional clarification.

Our study demonstrated the inaccuracy of the current definition of QMMD and suggested that the presence or absence of genetic mutations may be helpful for accurately diagnosing QMMD. However, no clinical variables showed significant associations with the presence or absence of gene mutations, as shown in Table 3, warranting further investigations in a larger population of patients. A limitation of this study is that the overall sample size was small due to the low prevalence of the disease, resulting in limited statistical studies. ${ }^{25,26}$ Thus, large-scale studies in a larger sample population are warranted.

Our subsequent short-term focus is to investigate whether the effect of different medical treatments on disease groups, for which those medical treatments are effective among the underlying disease with QMMD, differs based on the presence or absence of the RNF213 c.14576G $>\mathrm{A}$ mutation.

\section{Conclusions}

Our findings suggest that the RNF213 c.14576G $>\mathrm{A}$ mutation might be a useful marker in contributing to the accurate diagnosis of QMMD, and that it may, therefore, aid in the selection of appropriate treatment strategies for some patients with QMMD as well as accurate prediction of disease conditions.

\section{Acknowledgments}

This work was supported by JSPS KAKENHI Grant-in-Aid for Scientific Research (C) number 18K(K)09008.

\section{References}

1. Tominaga T, Suzuki N, Miyamoto S, Koizumi A, Kuroda S, Takahashi JC, et al. Recommendations for the management of moyamoya disease: a statement from research committee on spontaneous occlusion of the circle of Willis (moyamoya disease) [2nd edition]. Surg Cerebral Stroke. 2018;46(1):1-24.

2. Czartoski T, Hallam D, Lacy JM, Chun MR, Becker K.

Postinfectious vasculopathy with evolution to moyamoya syndrome. J Neurol Neurosurg Psychiatry. 2005;76(2):256-259.

3. Im SH, Oh CW, Kwon OK, Kim JE, Han DH. Moyamoya disease associated with Graves disease: special considerations regarding clinical significance and management. J Neurosurg. 2005;102(6):1013-1017.

4. Matano F, Murai Y, Adachi K, Kitamura T, Teramoto A. Pathophysiology and management of intracranial arterial stenosis around the circle of Willis associated with hyperthyroidism: case reports and literature review. Neurosurg Rev. 2014;37(2):347-356.

5. Miyatake S, Miyake N, Touho H, Nishimura-Tadaki A, Kondo Y, Okada I, et al. Homozygous c.14576G >A variant of RNF213 predicts early-onset and severe form of moyamoya disease. Neurology. 2012;78(11):803-810.

6. Liu W, Morito D, Takashima S, Mineharu Y, Kobayashi H, Hitomi T, et al. Identification of RNF213 as a susceptibility gene for moyamoya disease and its possible role in vascular development. PLoS One. 2011;6(7):e22542.

7. Kamada F, Aoki Y, Narisawa A, Abe Y, Komatsuzaki S, Kikuchi A, et al. A genome-wide association study identifies RNF213 as the first Moyamoya disease gene. J Hum Genet. 2011;56(1):34-40.

8. Rosser TL, Vezina G, Packer RJ. Cerebrovascular abnormalities in a population of children with neurofibromatosis type 1 . Neurology. 2005;64(3):553-555.

9. Ishikawa T, Houkin K, Yoshimoto T, Abe H. Vasoreconstructive surgery for radiation-induced vasculopathy in childhood. Surg Neurol. 1997;48(6):620-626.

10. Jea A, Smith ER, Robertson R, Scott RM. Moyamoya syndrome associated with Down syndrome: outcome after surgical revascularization. Pediatrics. 2005;116(5):e694-e701.

11. Scott RM, Smith JL, Robertson RL, Madsen JR, Soriano SG, Rockoff MA. Long-term outcome in children with moyamoya syndrome after cranial revascularization by pial synangiosis. J Neurosurg. 2004;100(2)(Suppl Pediatrics):142-149.

12. Kim SJ, Heo KG, Shin HY, Bang OY, Kim GM, Chung CS, et al. Association of thyroid autoantibodies with moyamoyatype cerebrovascular disease: a prospective study. Stroke. 2010;41(1):173-176.

13. Moteki Y, Onda H, Kasuya H, Yoneyama T, Okada Y, Hirota $\mathrm{K}$, et al. Systematic validation of RNF213 coding variants in Japanese patients with moyamoya disease. J Am Heart Assoc. 2015;4(5):e001862.

14. Watanabe A, Karasugi T, Sawai H, Naing BT, Ikegawa S, Orimo H, Shimada T. Prevalence of c.1559delT in ALPL, a common mutation resulting in the perinatal (lethal) form of hypophosphatasia in Japanese and effects of the mutation on heterozygous carriers. J Hum Genet. 2011;56(2):166-168.

15. Miyawaki S, Imai H, Shimizu M, Yagi S, Ono H, Nakatomi $\mathrm{H}$, et al. Genetic analysis of RNF213 c.14576G $>$ A variant in nonatherosclerotic quasi-moyamoya disease. J Stroke Cerebrovasc Dis. 2015;24(5):1075-1079.

16. Morimoto T, Mineharu Y, Kobayashi H, Harada KH, Funaki T, Takagi Y, et al. Significant association of the RNF213 p.R4810K polymorphism with quasi-moyamoya disease. $J$ Stroke Cerebrovasc Dis. 2016;25(11):2632-2636.

17. Phi JH, Choi JW, Seong MW, Kim T, Moon YJ, Lee J, et al. Association between moyamoya syndrome and the RNF213 c. $14576 \mathrm{G}>\mathrm{A}$ variant in patients with neurofibromatosis Type 1. J Neurosurg Pediatr. 2016;17(6):717-722.

18. Chong PF, Ogata R, Kobayashi H, Koizumi A, Kira R. Early onset of moyamoya syndrome in a Down syndrome patient with the genetic variant RNF213 p.R4810K. Brain Dev. 2015; 37(8):822-824.

19. Zhang Q, Liu Y, Yu L, Duan R, Ma Y, Ge P, et al. The association of the RNF213 p.R4810K polymorphism with quasimoyamoya disease and a review of the pertinent literature. World Neurosurg 2017;99:701-708.e1.

20. Nomura S, Akagawa H, Yamaguchi K, Ishikawa T, Kawashima A, Kasuya $\mathrm{H}$, et al. Rare and low-frequency variants in RNF213 confer susceptibility to moyamoya syndrome associated with hyperthyroidism. World Neurosurg. 2019;127: e460-e466.

21. Miyawaki S, Imai H, Shimizu M, Yagi S, Ono H, Mukasa A, et al. Genetic variant RNF213 c.14576G $>$ A in various phenotypes of intracranial major artery stenosis/occlusion. Stroke. 2013;44(10):2894-2897.

22. Hiraide T, Kataoka M, Suzuki H, Aimi Y, Chiba T, Isobe S, et al. Poor outcomes in carriers of the RNF213 variant (p.Arg4810Lys) with pulmonary arterial hypertension. $J$ Heart Lung Transplant. 2020;39(2):103-112.

23. Morimoto T, Mineharu Y, Ono K, Nakatochi M, Ichihara S, Kabata R, et al. Significant association of RNF213 p.R4810K, a moyamoya susceptibility variant, with coronary artery disease. PLoS One. 2017;12(4):e0175649. 
24. Cao Y, Kobayashi H, Morimoto T, Kabata R, Harada KH, Koizumi A. Frequency of RNF213 p.R4810K, a susceptibility variant for moyamoya disease, and health characteristics of carriers in the Japanese population. Environ Health Prev Med. 2016;21(5):387-390.

25. Greenland S, Schwartzbaum JA, Finkle WD. Problems due to small samples and sparse data in conditional logistic regression analysis. Am J Epidemiol. 2000;151(5):531-539.

26. Peduzzi P, Concato J, Kemper E, Holford TR, Feinstein AR. A simulation study of the number of events per variable in logistic regression analysis. J Clin Epidemiol. 1996;49(12): 1373-1379.

\section{Disclosures}

The authors report no conflict of interest concerning the materials or methods used in this study or the findings specified in this paper.

\section{Author Contributions}

Conception and design: Murai. Acquisition of data: Murai, Shirokane, Matano, Tsukiyama, Baba, Nakagawa, Tamaki, Mizunari, Tanikawa. Analysis and interpretation of data: Ishisaka, Watanabe. Drafting the article: Ishisaka. Critically revising the article: Murai. Reviewed submitted version of manuscript: Morita. Approved the final version of the manuscript on behalf of all authors: Ishisaka. Administrative/technical/material support: Murai, Morita. Study supervision: Murai, Morita.

\section{Correspondence}

Eitaro Ishisaka: Nippon Medical School, Tokyo, Japan. e-ishisaka@nms.ac.jp. 